\title{
Empresas familiares de inmigrantes en Barcelona y Montevideo
}

\author{
Paola Martinez Pérez \\ GEDIME (Grupo de Estudios de Inmigración y Minorías Étnicas) \\ paola.martinez@elogia.net
}

\begin{abstract}
Resumen
El objetivo de este trabajo es observar las diferentes dimensiones que adquiere la familia en las empresas familiares de inmigrantes. En este caso, la propuesta metodológica es analizar, de forma comparativa, la realidad empresarial consolidada del colectivo gallego en Montevideo con las empresas familiares de inmigrantes de reciente creación en el área metropolitana en Barcelona.

Palabras clave: inmigración, empresa, familia.

Abstract

The objective of this work is to observe the different dimensions taken on by the family in immigrant family firms. In this case, the methodological proposal is to use qualitative empirical evidence to compare established Galician enterprises in Montevideo with recently created immigrant family firms in the metropolitan area of Barcelona.
\end{abstract}

Keywords: immigration, firm, family. 


\section{Empresas familiares de inmigrantes‡ una definición}

La empresa familiar regentada por inmigrantes se caracteriza por las diferentes dimensiones que adquiere la familia en la propia actividad de la empresa cuando ésta se concibe en un contexto migratorio. En primer lugar, es preciso destacar que la familia es un factor diferencial a la hora de estudiar la iniciativa empresarial de los inmigrantes. En este sentido, la dimensión familiar actúa, dentro de las empresas familiares de inmigrantes, fundamentalmente de dos maneras: $a$ ) como un recurso étnico; $b$ ) como un recurso de clase. Además, debido a la desigualdad de géneros, se debe tener en cuenta un tercer factor: la división sexual del trabajo en la empresa y en la familia. La red familiar se puede considerar como una fuente de recursos - materiales y no materiales- que funciona casi como un «factor de producción» que se comporta de manera especial. Sin embargo, la red familiar no se presenta como una variable unidimensional, sino que, en su imbricación con la empresa - la empresa familiar-, manifiesta su relación en diferentes espacios.

En primer lugar, la familia es una fuente de conocimiento en el ámbito empresarial. Cuando la empresa familiar pasa a manos de una nueva generación, no sólo se transmite el valor material de sus bienes, sino que los herederos también obtienen el bagaje empresarial de su familia. En segundo lugar, la familia, sobre todo en un contexto migratorio, se convierte en un recurso de mano de obra barata o gratuita dentro de la empresa familiar. En tercer lugar, la división sexual del trabajo en la empresa familiar varía con la inmigración. El hecho migratorio determina nuevos patrones institucionales y culturales que se van combinando de forma dinámica con factores ya aprendidos en la sociedad de origen, dando lugar a nuevas formas de conciliación de roles según el género dentro de la empresa familiar y la familia. Estas dimensiones distinguen a las empresas familiares de inmigrantes de la empresa familiar autóctona actual, pero también de los «negocios étnicos» definidos en la literatura anglosajona (Kloosterman, 2000). Las nuevas empresas familiares cumplen algunos de los requisitos definitorios impuestos por las teorías de la empresa familiar, si bien es cierto que en otros aspectos presentan una relación clara con las corrientes teóricas que se han impuesto en la literatura de los negocios étnicos.

Para abordar el objeto de estudio, se construyó un marco teórico interdisciplinario en torno a las empresas familiares de inmigrantes. En ese modelo se combinan dos disciplinas: la sociología y la economía de la empresa. En concreto, se abordan las teorías sociológicas de los negocios étnicos, que se centran en la perspectiva de la incrustación mixta y en los estudios sobre la perspectiva de género en los negocios étnicos; y las teorías de la empresa familiar, que se ajustan a las teorías que versan sobre la empresa 
familiar. Por último, se utiliza la sociología de la familia, desde una perspectiva feminista crítica.

En el ámbito metodológico, se realizó un trabajo de campo que consistió en entrevistas en profundidad semidirigidas con emprendedores de origen inmigrante. ${ }^{1}$ Los datos obtenidos en el caso catalán para los empresarios no comunitarios se han analizado comparativamente con los patrones observados en el trabajo de campo obtenido en Montevideo, donde se han estudiado los empresarios gallegos.

\section{La iniciativa empresarial en un contexto migratorio: corrientes teóricas de los negocios étnicos y de la empresa familiar}

Los inmigrantes encuentran una serie de barreras a la hora de acceder al mercado de trabajo formal. Por lo general, los puestos de trabajo vacantes que ocupan los inmigrantes en las sociedades receptoras están marcados por una baja cualificación profesional. De esta manera, podemos agrupar las actividades en se emplean los trabajadores inmigrantes en dos categorías. La primera categoría comprende aquellas actividades manuales que requieren un gran esfuerzo físico y un bajo nivel de estudios. Dentro de esta categoría se incluyen las actividades relacionadas con el trabajo agrícola, la construcción, la minería, etc. La segunda categoría responde a nuevas profesiones que surgen al calor de la demanda que se crea en las sociedades modernas postindustriales: las tareas reproductivas o de cuidado del hogar y la familia, que eran una labor no reconocida socialmente y realizada por mujeres, quedan desatendidas cuando todos los miembros de la familia se incorporan al mercado laboral general y no hay ningún adulto dentro del entorno familiar que se pueda ocupar de ellas. ${ }^{2}$

Así, la oferta laboral a la que acceden los extranjeros se halla, en la mayor parte de los casos, restringida a una serie de puestos de trabajo caracterizados por la terciarización, una clara vocación asistencial y, en definitiva, la precariedad laboral. Estos

1 La investigación se ha estructurado en tres momentos o fases. La génesis de esta investigación se sitúa en febrero de 2003, cuando comienza la experiencia exploratoria a través de la participación en el proyecto de investigación desarrollado por el GEDIME bajo el título «Negocis ètnics a Catalunya». En esta primera fase, se realizaron cinco entrevistas en Barcelona y una en Tarragona. En total, se entrevistó a tres hombres y tres mujeres mediante un cuestionario breve de aspectos descriptivos relacionados con el negocio. En una segunda fase, con un proyecto previamente diseñado, se realizó otra serie de entrevistas en Sabadell y Castelldefels. Se trata de siete entrevistas con empresarios inmigrantes y una entrevista con una informante privilegiada, enfocadas a partir de las preguntas iniciales de investigación. La tercera parte comprende el trabajo de campo realizado entre septiembre y diciembre de 2006 en Montevideo (Uruguay), donde se visitaron 25 empresas de propietarios gallegos.

2 Estas lagunas de la demanda laboral se cubren, cada vez más, con la mano de obra de mujeres trabajadoras e inmigrantes (Parella, 2003). 
servicios se han convertido en auténticos «nichos laborales»3 (Engelen, 2001) para los trabajadores ocupados en ellos.

Una de las principales razones que esgrimen algunos autores para explicar la iniciativa empresarial del colectivo inmigrante es el proceso de desclasamiento relacionado con la inmigración (Wright y Ellis, 2001). Este proceso se debe a que las condiciones institucionales, económicas e, incluso, sociales de los países receptores dificultan que estas personas puedan mantener la misma cualificación profesional y el mismo nivel de clase social que en su país de origen.

Si estudiamos el fenómeno de la creación de empresas como un «mercado de empresariado» (Kloosterman, 2000; Kloosterman y Rath, 2001), la oferta estará compuesta por aquellos inmigrantes emprendedores que quieran intentar establecer su propio negocio. La demanda será la propia estructura de oportunidades, es decir, estará constituida por aquellos huecos de mercado donde exista potencialmente la oportunidad de negocio.

Según Kloosterman, esta demanda está compuesta principalmente por empresas a pequeña escala, con bajas necesidades tecnológicas, pertenecientes al sector servicios y que se sitúan espacialmente en el entorno del cliente. En una primera tipología, distingue entre servicios personales - como restaurantes, servicios de guardería, peluquerías, sastrerías o servicio doméstico-y otros servicios - como seguridad, limpieza general y servicios de mensajería-. Todos estos servicios se caracterizan por el contacto cara a cara, porque se producen en el acto y porque, generalmente, no se pueden importar.

Los servicios personales se suelen situar en nichos de mercado abandonados por los autóctonos por su baja rentabilidad y las duras condiciones laborales. Son, normalmente, actividades tradicionales que han quedado relegadas a un segundo plano por la aparición de grandes empresas que compiten con unas mejores condiciones de mercado. Sin embargo, estos servicios de proximidad han sido el sector donde muchos inmigrantes han desarrollado su iniciativa empresarial (Kloosterman, 2000; Kloosterman y Rath, 2001; Solé y Parella, 2005; Waldinger, Aldrich y Ward, 1990).

El segundo tipo de actividades responde a la necesidad creada por la sociedad postindustrial. Antiguamente, la unidad familiar se había sustentado, por una parte, en la actividad laboral dentro de la economía formal del padre de familia y, por otra, en la actividad reproductiva que la mujer realizaba dentro del hogar. En la sociedad postindustrial, esta forma de unidad familiar se transforma en hogares donde tanto el hombre como la mujer trabajan. Así, se crea una nueva necesidad dentro del mercado de servicios: los servicios reproductivos, que suplen el rol de la mujer ocupada en las tareas

3 Se han llegado a definir como «nichos laborales», porque son ocupaciones muy intensivas en cuanto a desgaste físico, no reconocidas socialmente (Beck-Gernsheim, 2003) y donde las oportunidades para desarrollar otras actividades más cualificadas o ascender son remotas. 
del hogar y el cuidado de niños y ancianos. Por otra parte, en las sociedades modernas, existen claras deficiencias en el modelo de Estado de Bienestar para poder hacer frente a las nuevas necesidades asistenciales de las familias y los individuos.

En el ámbito académico estadounidense y europeo, han surgido diferentes teorías sobre los negocios étnicos que pretenden identificar las variables que influyen en las diferencias emprendedoras que existen entre los distintos grupos étnicos (Riesco, 2004). Solé y Parella (2005) distinguen cuatro grupos de teorías: a) «las teorías de carácter étnico-cultural (teorías culturales)», b) «las teorías que identifican el auge de los negocios étnicos como una consecuencia directa de las desventajas padecidas por los inmigrantes en su inserción en los mercados de trabajo», c) las teorías menos deterministas o interactivas, que hacen referencia a las estructuras de oportunidad (recursos de clase y recursos étnicos) que rodean a los empresarios inmigrantes (Waldinger, Aldrich y Ward, 1990) y, por último, d) la teoría de la mixed embeddedness, o incrustación mixta, gestada en Europa a finales de los años noventa y desarrollada por Kloosterman y sus colaboradores (Barret, Jones y McEvoy, 2001). ${ }^{4}$

En estos momentos, las empresas familiares de inmigrantes en España se encuentran todavía en una primera fase - en la primera generación-, de manera que, en la mayor parte de los casos, no se sabe si habrá sucesión o, si la hay, qué pasará con la siguiente generación. En cualquier caso, se puede establecer un paralelismo entre esta realidad empresarial en un contexto de inmigración y lo que fue la historia empresarial española durante el pasado siglo en los países de «emigración», como lo fue Uruguay, donde aparecieron grandes emprendedores, en su mayoría gallegos, que llevaron a cabo proyectos de empresa familiar muy importantes. Estos empresarios demostraron su éxito superando la etapa de madurez del negocio (Gallo, Cuatrecasas, Arriola y Casado, 1998) y el paso de los años (Gallo, 1998). En consecuencia, hay que esperar para ver el desarrollo de estas empresas familiares de inmigrantes que han nacido recientemente y cuyo futuro, como el de cualquier empresa familiar que emerge, aún es incierto.

\footnotetext{
4 Por lo que respecta a las aproximaciones teóricas, en este caso se opta por seguir la perspectiva de la incrustación mixta. Este modelo consiste en explicar las diferencias entre grupos desde una aproximación que enfatiza la importancia de las redes coétnicas sociales y culturales en combinación con otra batería de factores institucionales y económicos para determinar las causas que llevan a estos inmigrantes a crear sus propios negocios familiares. Este enfoque hace mención a dos tipos de factores explicativos. El primer grupo de factores se refiere al contexto institucional y económico donde se crean estas empresas de propiedad inmigrante. El segundo grupo define las particularidades vinculadas a la comunidad y a las redes familiares de las personas inmigradas que deciden ser empresarias.
} 


\section{Configuración de la familia en la inmigración y en la empresa familiar: corrientes teóricas de la sociología de la familia}

Según Flaquer (1998), la familia es la agrupación humana por antonomasia y la más elemental de todas. En todas las sociedades existe un elemento común‡ la necesidad de construir un espacio donde el individuo pueda desarrollarse (Green y Johns, 1973). Este espacio se crea en la familia, que normalmente está compuesta por una red de miembros unidos por relaciones de consanguinidad. De todas maneras, la concepción de familia varía según la época, la raza, la religión o la clase social. En algunas culturas, la familia está restringida a los miembros más allegados, mientras que en otras, como ocurre en algunos países africanos y asiáticos (Thumerelle, 1997), pueden considerarse familia incluso personas que no son consanguíneas, pero que mantienen un fuerte vínculo de proximidad.

El modelo de familia que domina en la cultura occidental es el de la familia nuclear. La familia nuclear tipo está constituida por dos generaciones de miembros unidas por una relación de parentesco y relaciones afectivas. En estas familias, los adultos se hallan unidos por medio de la institución del matrimonio. Esta tipología de familia está tan extendida en la sociedad actual que, cuando se hace referencia a la familia, se sobreentiende que se habla de la familia nuclear.

Desde todos los ámbitos, no obstante, se habla de una nueva sociedad donde la familia está adoptando formas diferentes y las relaciones vecinales o con la comunidad están perdiendo el peso que habían alcanzado años atrás (Beck-Gernsheim, 2003). Como explica Flaquer (1998), es importante no circunscribir la familia al ámbito privado, pues en ella hay mucha más política de lo que creemos. Un ejemplo de este matiz político y social se puede encontrar en la evolución paralela de familia e inmigración.

\subsection{La familia en un contexto migratorio}

La familia no fue un elemento vinculado invariablemente a la inmigración. Los primeros movimientos migratorios de la época actual respondían a un ajuste entre oferta y demanda del factor productivo trabajo. En la Revolución Industrial, los trabajadores ${ }^{5}$ acudían allí donde los empresarios necesitaban mano de obra para buscar un puesto de trabajo o mejores condiciones laborales. Este tipo de emigración era una aventura individual cuyo principal objetivo era la acumulación de bienes materiales para enviar a sus

5 Hasta bien entrado el siglo $\mathrm{xx}$, la mujer no ha tenido un papel reconocido por la sociedad dentro del mundo laboral como asalariada, de manera que la emigración económica era tradicionalmente masculina. 
familias en el país de origen, sin planear — al menos en principio- un establecimiento duradero en el nuevo lugar de trabajo.

En Estados Unidos, a partir de la década de 1960, esta tendencia se rompió (Sassen, 1993). Por una parte, se produjo un cambio en los valores sociales postindustriales (Sassen, 1991). El inmigrante económico no sólo buscaba mejorar su situación en cuanto a la acumulación de riqueza, sino que, una vez transcurrido un margen de tiempo, intentaba trasladar a su familia desde el país de origen a la sociedad de destino. ${ }^{6}$ Esta tendencia se vio reforzada en el ámbito estadounidense, y también en muchos países occidentales - receptores de inmigración-, por una legislación orientada a la reunión de la familia (Castles y Kosack, 1984).

Las políticas de inmigración en cuyo marco nace la figura de la reagrupación familiar eligen esta posibilidad no sólo para satisfacer la necesidad del inmigrante de reconstruir su entorno relacional en el país receptor, sino también para aprovechar el poder de atracción sobre el consumo que se obtiene cuando la familia se reúne en un nuevo país (Aranda Gallego, 2003; Aparicio y Tornos, 2000; Barrada, 2003; Segura Lucas, 2004). En la actualidad, la mayor parte de los países receptores netos de trabajadores extranjeros desarrollan una política de inmigración que protege la unidad familiar. En el caso español, el inmigrante, independientemente de su género, tiene la posibilidad de reagrupar a sus familiares en el momento en que se encuentre en una situación regular y estable.

Los familiares reagrupados por inmigrantes en España se enfrentan a su vez a un mercado laboral muy restrictivo debido a las políticas migratorias. En muchos casos, no obtienen permiso para trabajar, desconocen el idioma, y el género les dificulta aún más su acceso al mercado laboral. En estos casos, si ya existe una empresa familiar, su aportación laboral a ella se prefiere a las ofertas de trabajo externas e, incluso, supone la única posibilidad de trabajar — al borde de la legalidad — (Kloosterman y Rath, 2001; Carrasco, 2003).

En este sentido, también hay que destacar que los inmigrantes suelen seguir trayectorias de vida - y por tanto, también laborales - conforme a patrones colectivos; es decir, se apoyan en la colectividad, en «los suyos», para desarrollar sus carreras de migración (migration careers) (Stillwell y Congdon, 1991) en el país de destino. Algunos autores han destacado en sus investigaciones la importancia de los enclaves (Riesco, 2004), y lo cierto es que muchos colectivos, al emigrar, se sitúan no sólo junto a sus connacionales, sino allí donde se encuentran su familia y sus vecinos en el nuevo país.

6 En el caso de las mujeres - entre finales del siglo xix y principios del siglo $\mathrm{xx}$-, la emigración era una estrategia económica - laboral一, pero también una estrategia familiar. SINKE, SUZANNE M. (2006). «Gender and Migration: Historical Perspectives», International Migration Review, 40 (1), pp. 82-103. 


\subsection{La división sexual del trabajo en la familia y en la empresa familiar}

Según Brunet y Alarcón (2004), se puede decir que la empresa, frente a lo que habitualmente se piensa, es un ente social y sexuado. En las empresas familiares, la dimensión familiar de la empresa está marcada por patrones que establecen una división sexual del trabajo. La división sexual del trabajo desde el punto de vista micro - en el seno de la empresa y en la familia - distribuirá las tareas o roles en la familia según el género. Por otra parte, en una esfera macro, se da una división internacional del trabajo que diferencia las tareas que se asignan a las mujeres inmigrantes de las tareas atribuidas a las mujeres autóctonas - supuestamente mejor cualificadas - (Brunet y Alarcón, 2004; Parella, 2003).

En un contexto migratorio, las condiciones que rodean a la familia influirán de diferentes formas. En primer lugar, en una sociedad multicultural - y receptora de inmigración - convivirán diferentes modelos de familia. En cada tipo de familia, las divisiones de tareas según el género de sus miembros serán diferentes para cada comunidad. ${ }^{7}$ En segundo lugar, la familia sufre una serie de transformaciones en la inmigración. Muchas veces, la unidad familiar se escinde entre el país de origen y el país de acogida. Así, los inmigrantes, en ocasiones, no disponen de la red familiar completa en el país donde se establecen. Esta situación lleva a muchas mujeres inmigrantes - que asumen las tareas reproductivas en solitario- a no poder hacerse cargo de sus hijos cuando tienen un trabajo por cuenta ajena, pues no disponen del apoyo de la red familiar, y por ello buscan soluciones alternativas, como la autoocupación o dejar a los hijos con la familia en la sociedad de origen. En tercer lugar, la división del trabajo no sólo distingue entre masculino y femenino. Así, lo femenino se halla fragmentado por otras variables transversales como la raza, la clase social, las características étnicas o la edad. De este modo, las mujeres inmigrantes en nuestro país reproducen el rol de género como cuidadoras «suplentes» de las mujeres de clase media que han abandonado la esfera doméstica de las responsabilidades reproductivas (Parella, 2003).

Los patrones propuestos por Parsons dominaron en la teoría social durante años. Su propuesta idealizaba la división sexual del trabajo como sistema de conciliación entre el mundo laboral y las responsabilidades reproductivas dentro del hogar (Murillo, 1996; Parella y Samper, 2007).

Cuando, tras la Revolución Industrial, se produjo la salida del trabajo de la esfera doméstica - progresivamente y primero entre las clases altas - se comenzó a valorar el aislamiento diario en un espacio de privacidad tras la jornada laboral. Además,

7 La comunidad determina el modelo de familia. En este caso, el modelo de familia vendrá definido por la cultura, la religión, los valores sociales, los factores institucionales, etc. (Curran, Shafer, Donato y Garip, 2006). 
existía una gran inquietud sobre la incorporación de la mujer al mundo laboral. Se creía que este factor, junto con el avance tecnológico, acabaría debilitando la posición hegemónica del hombre dentro de la oferta de trabajo. Sin embargo, al comienzo de la Revolución Industrial, el trabajo fabril no distinguía entre género o edad. De este modo, fue a través de la especialización como los hombres consiguieron controlar la competencia del trabajo femenino, puesto que ese «plus» formativo sólo estaba al alcance de los hombres, por lo menos socialmente. Como consecuencia, el trabajo de la mujer tenía mero carácter suplementario o de ayuda, y, en todo caso, era algo excepcional. Fue a través de esta pérdida de protagonismo en la sociedad como las mujeres se «ocultaron en la trastienda» de los negocios familiares.

En el modelo de conciliación propuesto por Parsons (Alberdi, 1996; Parsons, 1982), se señalan ciertas cualidades o aptitudes para el desarrollo de determinados roles según el género. Así, se apunta que, mientras que los hombres están mejor preparados para asumir un rol instrumental, las mujeres desarrollan a la perfección un rol expresivo. Al hombre, por tanto, le corresponde la salida al exterior, el mantenimiento económico de la familia y la representación social de ésta. Mientras tanto, a la mujer, preparada ya fisiológicamente para tener hijos, se le atribuía el cuidado de los niños y de los adultos (Alberdi, 1996). En la vida dentro de la empresa familiar, esto se traducía en un rol visible del empresario varón como emprendedor y de los hombres de la familia empleados en la empresa familiar, y en un papel también importante, pero oculto, secundario y no reconocido de las mujeres.

Pese a la desigualdad, se creía que esta distribución era, precisamente, la que en ocasiones hacía posible el funcionamiento de la empresa familiar y la familia. Actualmente, el modelo familiar patriarcal sobre el que se construyeron muchas de las empresas familiares españolas más añejas ha entrado en crisis (Osborne, 2004) y ha dado paso a una amplia tipología de nuevas formas organización de la conciliación en la familia (Beck-Gernsheim, 2003). De este modo, en la actualidad existen tres teorías de explicación alternativas al patrón parsoniano sobre el reparto de tareas según el sexo; es decir, modelos que intentan explicar por qué y cómo funciona la división sexual del trabajo en la sociedad, que se han ido combinando según la época histórica y los diferentes tipos de sociedad: a) la nueva economía de la familia, $b$ ) la perspectiva institucionalista y marxista y c) el modelo de producción/reproducción (Brunet y Alarcón, 2004).

Una de las principales diferencias entre las empresas familiares de inmigrantes en España y las empresas familiares de los propios españoles es, precisamente, la estructura familiar, que está vinculada a los factores socioculturales a los que hace referencia la teoría de la incrustación mixta (Kloosterman, 2000; Kloosterman y Rath, 2001; Kloosterman, Van der Leun y Rath, 1999). 
El empresariado inmigrante responde a diferentes patrones familiares. El modelo de familia está determinado por elementos culturales — como la religión o la clase social- y por elementos coyunturales - como el hallarse en un contexto migratorio(Parella y Samper, 2007). Estos dos elementos hacen que las familias inmigrantes que sustentan las empresas familiares respondan normalmente a patrones tradicionales y conservadores. Por ejemplo, en el caso de las empresas familiares de gallegos en Montevideo, el peso del patriarcalismo sigue siendo muy fuerte y está plenamente aceptado. En este caso, es posible que las propias características del entorno migratorio hagan necesaria la existencia de un modelo que sea admitido por ambas partes, que evite el conflicto y que logre la supervivencia de la empresa.

En general, la familia dentro de la empresa familiar desempeña dos papeles fundamentales. En primer lugar, es una fuente de mano de obra barata o gratuita. Muchas veces, los familiares que trabajan en el negocio familiar no lo hacen a tiempo completo, sino que lo combinan con otros trabajos. Las condiciones laborales son muy precarias en la mayor parte de los casos: largas jornadas de trabajo todos los días de la semana, tareas de gran desgaste físico, sin contrato laboral y sin vacaciones estipuladas y pagadas. En segundo lugar, la familia del emprendedor, en muchos casos, es la que le ayuda a poner en marcha su negocio proporcionándole la financiación o el aval necesario para obtener el dinero necesario en un medio externo (Barret, Jones y McEvoy, 2001; Haberfelliner, 2000; Leung, 2000).

En el caso de las mujeres que trabajan en la empresa familiar, a pesar de que existan condiciones que propician la discriminación y la desigualdad, no hay que dejar de reconocer que, dependiendo del contexto, también les pueden proporcionar una serie de ventajas. En primer lugar, en la mayor parte de los casos, la inserción laboral dentro del negocio familiar requiere superar menos barreras - como el dominio del idioma, el poseer una titulación académica válida para ejercer su profesión en el país, etc. - que la incorporación al mercado laboral general. En segundo lugar, la gestión del tiempo de trabajo en la empresa y del trabajo reproductivo resulta más flexible, generalmente, cuando se trabaja bajo la supervisión familiar. En tercer y último lugar, mantener el patrón tradicional patriarcalista, donde el hombre es el sustentador principal de la unidad familiar, puede ser una opción para mujeres que tienen tareas reproductivas ineludibles a su cargo y pueden tener la posibilidad de trabajar desde casa (Solé y Parella, 2005).

Otro aspecto que caracteriza a las empresas familiares es la sucesión, un tema que ha sido tradicionalmente estudiado por la literatura sobre la empresa familiar desde el punto de vista jurídico (Gallo, 1998). El término «sucesión» se identifica con el fenómeno legal del traspaso de la empresa — dentro de la familia - de una generación a otra. Así mismo, la sucesión es uno de los elementos más importantes dentro de las 
definiciones estrictas de «empresa familiar», que entienden que la empresa debe pasar por la sucesión y permanecer un tiempo determinado en manos de la segunda o tercera generación - varía según las definiciones - para poder considerar que estamos ante una empresa familiar.

El hecho migratorio puede hacernos reflexionar, en un contexto transnacional, sobre el concepto de «sucesión» entendida en un sentido muy laxo, es decir, simplemente como la continuidad del negocio. De este modo, existirían tres tipos de «sucesión» en las empresas familiares de inmigrantes. El primer tipo es el clásico de la empresa familiar. Así, los dueños de los negocios estudiados más exitosos desean que sus hijos les sucedan al frente de la empresa. En este contexto, la empresa se convierte en un legado que les gustaría que perviviese dentro de la familia. Sin embargo, los profesionales liberales ven la sucesión como un proyecto no tan atractivo para sus congéneres.

El segundo tipo hace referencia al capital social adquirido en la familia. En algún caso, la sucesión en el contexto migratorio puede no ser una sucesión material ni limitada al ámbito local. Si el empresario emprende en la sociedad de acogida el mismo negocio que tenía su familia en la sociedad de origen, podemos considerar que éste es también un tipo de «suceder», de tomar el testigo de la tradición empresarial familiar, aunque sea a miles de kilómetros.

Por último, el fenómeno del abandono de pequeños negocios familiares por parte de los autóctonos — precisamente por la falta de «sucesión» propiamente dicha-, que pasan así a manos de empresarios inmigrantes - lo que se ha llamado «sustitución» (Solé y Parella, 2005; Barret, Jones y McEvoy, 2001)—, se convierte también en una especie de «sucesión» no familiar.

\subsection{La evolución de la presencia femenina en las empresas familiares de inmigrantes}

En primer lugar, se debe señalar que la literatura existente sobre empresariado étnico abordada desde una perspectiva de género aún es muy escasa (Solé y Parella, 2005; Kontos, 2000; Oso y Ribas, 2004). Para explicar este hecho, algunas autoras señalan que el fenómeno migratorio femenino se ha visto invisibilizado tradicionalmente tanto en la realidad social como en el mundo académico (Parella, 2003; Oso y Ribas, 2004). Así, Oso y Ribas explican la carencia de publicaciones referentes a la mujer inmigrante empresaria por una extensión de la invisibilización académica - reconocida - en el caso de la realidad que rodea a la mujer como inmigrante. Sin embargo, las mujeres 
han sido pilares fundamentales tanto en la creación de empresas familiares como en la contribución a su desarrollo y mantenimiento.

En cuanto al papel de la mujer en la empresa familiar, podemos distinguir los siguientes roles: $a$ ) empresarias, $b$ ) trabajadoras reconocidas y $c$ ) trabajadoras no reconocidas.

Las empresarias inmigrantes que crean una empresa familiar manejan las mismas estrategias que los hombres empresarios en cuanto a la utilización de los recursos de clase y recursos étnicos (Light y Gold, 2000). Sin embargo, debido a las reminiscencias del modelo parsoniano de división sexual del trabajo, las mujeres empresarias se ven obligadas a combinar familia y empresa de una forma quizás más intensiva que las trabajadoras por cuenta ajena. La flexibilidad horaria desempeña una doble baza: permite conciliar la vida familiar con la vida laboral (Solé y Parella, 2005), pero, por otra parte, puede llegar a constituir una vinculación permanente con las responsabilidades reproductivas (Oso y Ribas, 2004).

Las mujeres inmigrantes que participan en las empresas familiares como trabajadoras con un papel reconocido encuentran en la empresa familiar un «colchón» que les permite incorporarse al mundo laboral en el país de acogida con cierta facilidad, sin tener que enfrentarse a las posibles barreras que tenga en el mercado laboral por cuenta ajena. Por otra parte, disfrutan de las ventajas de la flexibilidad mencionada anteriormente para el caso de las mujeres empresarias. En este sentido, un mayor control sobre la gestión del tiempo les supondrá una facilidad extra para la conciliación entre empresa y familia, si bien es cierto que, en este caso, puede producirse una sobreextensión de las responsabilidades reproductivas bajo el argumento de la flexibilidad.

Por último, existe un grupo de mujeres que trabajan de manera no reconocida, como puntualizan Oso y Ribas (2004) en la investigación que realizan sobre el colectivo de mujeres marroquíes empresarias en Barcelona. Se trata de puestos de trabajo dentro de la empresa familiar que no son reconocidos ni jurídicamente, ni incluso, a veces, socialmente. Sin embargo, hay ocasiones en que estas mujeres forjan estrategias paralelas que les permiten alcanzar cierta posición de poder.

Algunas autoras, como Floya Anthias, han trabajado acerca de la explicitación de la contribución implícita femenina al desarrollo y sostenimiento de los negocios étnicos (Anthias, 2000). En un libro anterior, referenciado en Kontos (2000), Anthias analiza, desde una perspectiva de género, las estrategias de los hombres inmigrantes grecochipriotas en Londres. Estos inmigrantes emprendedores se situaban en un sector de negocios que comprendía, principalmente, el catering y las industrias textiles. La autora concluye que la familia de estos empresarios, así como las mujeres que pertenecían a la misma comunidad, eran la fuerza de trabajo base que mantenía el éxito de estas em- 
presas. Incluso observa estrategias de matrimonio encaminadas a mejorar la posición de las actividades empresariales masculinas.

Según Maria Kontos (2000), la invisibilidad que tiene la mujer dentro de las teorías y realidades de los negocios étnicos — como empresaria y trabajadora reconocida - se debe, en parte, a que el rol de la mujer se adscribe directamente a los recursos étnicos de que disfrutan los inmigrantes según las teorías culturalistas de negocios étnicos, como en la obra de Light (1972). Sin embargo, Kontos señala que, para el caso alemán, de las estadísticas extraídas del censo de autoempleados, cruzando las dimensiones de género y grupo étnico, se obtiene una sorprendente ratio de crecimiento de la iniciativa empresarial entre las mujeres inmigrantes: mientras que el número de mujeres inmigrantes empresarias creció desde 1985 hasta 1998 en un $119 \%$, el número de hombres inmigrantes empresarios aumentó, en el mismo período, un $86 \%$. Cuando compara la iniciativa empresarial de los inmigrantes con la de los propios alemanes para el período 1985-1998, observa que el autoempleo entre las mujeres alemanas ha crecido un 44\%, mientras que en el caso de los hombres alemanes sólo lo ha hecho en un 16\% (Kontos, 2000).

Según las investigaciones sobre la empresarización de las mujeres inmigrantes (Kontos, 2000; Oso y Ribas, 2004), existen tres formas distintas de que las mujeres inmigrantes puedan llevar a cabo sus proyectos empresariales. En primer lugar, a través de un proyecto propio; en este caso, la mujer decide hacerse empresaria y lleva a cabo un proyecto emprendedor «novedoso», en solitario o de forma societaria, en el que puede implicar al resto de la familia o a otros miembros de la comunidad. En segundo lugar, la mujer inmigrante puede ascender desde el puesto de asistente reconocida en el negocio familiar a un escalafón directivo de la empresa, a través de una asimilación de las funciones directivas. En tercer lugar, las mujeres asistentes no reconocidas dentro de las empresas familiares de inmigrantes experimentan una transformación en su rol laboral, normalmente acompañando al éxito del negocio familiar.

La decisión emprendedora de las mujeres inmigrantes en la economía y la sociedad receptoras no es siempre producto de la economy of need a la que hacen referencia algunos autores. En muchos casos, como constata Kontos (2000) en su investigación, la realización personal y el desarrollo de la creatividad son factores que empujan a estas mujeres a llevar a cabo y mantener su actividad empresarial.

La diferencia fundamental en cuanto a la inserción laboral —en cualquiera de los tres roles anteriormente mencionados - en la empresa familiar de las mujeres inmigrantes respecto a las mujeres autóctonas reside en la estrategia de conciliación. La conciliación del ámbito laboral y familiar de las mujeres inmigrantes está determinada por dos aspectos fundamentales: la red familiar y el acceso a las políticas sociales. Así, las mujeres inmigrantes deben conciliar la vida laboral y familiar en unas condiciones 
más restrictivas. Los recursos familiares disponibles para la conciliación se transforman en un contexto migratorio. La red familiar queda fragmentada entre el país de origen y el país de destino. Por tanto, las mujeres inmigrantes en pocas ocasiones pueden contar con estos recursos a la hora de diseñar sus estrategias de conciliación. La condición legal de las mujeres inmigradas también determina las diferencias en el acceso a las políticas sociales disponibles. De este modo, las mujeres inmigrantes que emprenden una empresa familiar o que se incorporan a empresas familiares en el país de destino se hallan influidas en sus decisiones por las responsabilidades reproductivas. Las limitaciones de la red familiar y las restricciones en la utilización de las políticas sociales llevan a estas mujeres a seguir estrategias de conciliación tradicionales, no tanto por cuestión cultural o ideológica, sino condicionadas por estos factores estructurales $(\mathrm{Pa}$ rella y Samper, 2007 ).

\section{La dimensión familiar en las empresas familiares en un contexto migratorio: algunos ejemplos extraídos del trabajo de campo en Barcelona y Montevideo}

Como se ha comentado anteriormente, la familia en la empresa familiar regentada por inmigrantes está presente en la empresa de tres maneras diferentes: como recurso de clase, como recurso étnico y en el marco de la división sexual del trabajo en el ámbito doméstico y empresarial. Estas tres opciones tienen reflejo en el trabajo de campo que comprende esta investigación. Para ilustrarlo, se muestran a continuación algunos ejemplos de los casos analizados.

En el caso de Josef, de origen senegalés, él y su mujer deciden montar un locutorio como salida laboral cuando ella llega de Senegal a España. En muchos casos, la formación de una empresa familiar lleva implícita una decisión de carácter familiar; es decir, se escoge el autoempleo o el empleo en una empresa de la familia para poder conciliar de forma más flexible la vida familiar y laboral (Solé y Parella, 2005; Kontos, 2000; Oso y Ribas, 2004; Anthias, 2000).

En esta ocasión, el control de la familia sobre el locutorio permite la «flexibilidad temporal» necesaria para combinar las tareas reproductivas y la vida laboral. Sin embargo, la «flexibilidad» y la disponibilidad de tiempo libre obtenida a partir del negocio se utilizan de forma diferente según el género de los copropietarios. Así, la flexibilidad, para ella, supone más tiempo para dedicarse a las tareas reproductivas y, para él, se traduce en la posibilidad de llevar a cabo más tareas de la «esfera pública» (Parsons, 1982). Este caso concuerda con las corrientes teóricas de la nueva economía de la familia. La mujer de Josef divide su tiempo disponible entre el trabajo en el locutorio, las ta- 
reas domésticas y el ocio. Sin embargo, Josef gestiona su tiempo de manera que o bien está trabajando fuera del hogar, o bien se dedica a actividades asociativas - tiempo de ocio- (Brunet y Alarcón, 2004). Todo ello se traduce, según la teoría institucionalista marxista, en que la división sexual del trabajo tradicional del mercado de trabajo es sustituida por una división sexual del tiempo de trabajo que asigna un tiempo completo para los hombres y un tiempo parcial para las mujeres (Lagrave, 1993). Su proyecto de futuro es cerrar el locutorio y emprender otro proyecto en Senegal. La idea del regreso está motivada por la preocupación que tienen ambos por la educación de sus hijas.

En el caso de José, un empresario dedicado al negocio de automóviles en Montevideo, la conciliación familiar no supuso un problema. Su mujer, que se dedicaba a las tareas reproductivas, $y$, después, la contratación de personas que se ocupaban de llevar los asuntos domésticos, le permitieron dedicarse por completo al negocio. En su caso, contó con recursos de mano de obra familiares, pues en los primeros tiempos del concesionario trabajaron con él dos de sus hermanos. Combina su labor empresarial con la participación en diferentes asociaciones empresariales y políticas. Actualmente, uno de sus hijos trabaja con él y espera continuar el negocio.

Para Ilda (una conocida chef hija de gallegos en Uruguay) o Rosa (una empresaria argentina en Barcelona), establecerse por cuenta propia cuando sus hijas eran pequeñas - estaba separada - fue su mejor manera de poder combinar el cuidado de sus hijas con el trabajo, aunque también contó con la ayuda de su madre.

«Me ayudaba mucho mi madre. Pero lo enfoqué un poco así de estar con ellas. Así es que ellas empezaron el colegio con tres y cuatro años. Las tuve conmigo. [... $] \mathrm{Me}$ permitía [combinarlo] porque lo hacía todo en casa, lo de las manualidades. Solamente la feria ésta de artesanía en las Ramblas era sábado y domingo, aunque iba mucho por la costa a vender en verano [... . Yo lo preparaba durante el invierno y luego les entregaba. Pero era muy cómodo para mí, sí, porque preparaba todo en casa.»

Sin embargo, para Rosa, la decisión de establecerse por cuenta propia o trabajar en el negocio familiar siempre fue una prioridad frente a las ofertas de trabajo por cuenta ajena. Ella lo atribuye, en parte, a la herencia del espíritu emprendedor de su padre, es decir, a los recursos de clase obtenidos a través de la familia:

«Sí, mi padre siempre ha sido un hombre muy emprendedor de negocios. En Argentina tenía una fábrica de calzados, que tenía muchas sucursales en toda Argentina. (Pः Porque me comentaste que llegó aquí, compró un hotel...) Sí, en Argentina también tuvo. Sí, creo que ha sido también porque mi padre me ha inculcado un poco el tema éste de ser autónomo, montar negocios.»

En cambio, su madre nunca tuvo un papel activo dentro de la empresa familiar, sino que ocupó un lugar con escasa visibilidad. Rosa reclama irónicamente el rol de «ama de casa» que ejerció su madre. Aunque ser empresaria le satisface profundamente, recono- 
ce que el modelo parsoniano de división sexual del trabajo proporcionaba una mayor «tranquilidad» a las mujeres.

«Mi mamá no. (Se ríe y bromea a continuación) Mi mamá... A veces le digo: «Me gustaría haber nacido en tu generación para estar más tranquila»»»

En cuanto a la unidad familiar, sus hijas se han independizado y no tienen otras cargas familiares. Ella y su marido se combinan tanto en las tareas domésticas como en la atención del negocio.

Por otra parte, tanto a Ilda como a Rosa les gustaría que, en un futuro, sus hijas siguieran con la actividad de la empresa. En el caso de Ilda es casi un hecho, pues su única hija trabaja ya codo a codo con ella en la empresa de catering que dirigen. Las dos hijas de Rosa, en cambio, ejercen otras profesiones no relacionadas con la hostelería, aunque ella mantiene la esperanza de convencerlas.

Velina es una mujer de origen ruso que reside en Barcelona y tiene cuatro hijos. Desde que tiene la empresa de fachadas verticales junto con su marido, ha podido conciliar mejor el trabajo reproductivo con su trabajo de comercial. Anteriormente, trabajaba por cuenta ajena en un negocio de hostelería y no podía permitirse ningún tipo de flexibilidad.

«Cuando yo tengo la empresa sí que es más fácil. Porque si está enferma o... yo qué se, hay excursiones..., puedo combinar. Puedo llamar por teléfono, puedo retrasar visita o lo que sea. Pero cuando trabajas con jefe... casi que no. Por ejemplo, si el niño está enfermo, él (su antiguo jefe) no me dejaba ir al cole a recogerlo.»

Ella y su marido comparten las tareas de la casa. La flexibilidad en el horario ha permitido a Velina apuntarse a diferentes cursos: obtención del permiso de conducir, formación empresarial, catalán, etc.

En el caso de Solange, una mujer argentina que llegó hace cinco años a Barcelona, ella y su marido no tienen hijos, por lo que no existen cargas familiares. La decisión de establecer su propio estudio de arquitectura se basa en razones laborales. Ella prefiere trabajar por su cuenta a trabajar por cuenta ajena. Su estudio de arquitectura comparte el espacio con el taller de cuchillero de su marido.

Ana, una odontóloga que vino de Argentina a Barcelona con su marido y sus hijas, no pudo montar la clínica dental hasta que sus hijas fueron mayores (por razones de financiación, etc.). En la actualidad, sus tres hijas trabajan con ella. Dos de ellas son dentistas y pueden conciliar esta actividad en el negocio familiar con otros trabajos como dentistas que realizan fuera de la empresa familiar. Valora positivamente su trayectoria en la clínica — no le gusta utilizar la palabra «empresaria»—, que de hecho ha sido, y continúa siendo, el medio de vida de la unidad familiar:

«Ha sido mi medio de vida, el medio de vida de toda la familia, porque luego mi marido dejó de trabajar en la Autónoma, no por gusto, sino porque ya no le renovaron 
los contratos. Luego tuvo trabajos muy esporádicos. La solvencia de la familia y todo se basó en la clínica. El llevar adelante la casa, los estudios de mis hijas, todo lo que fue la vida hasta ese momento, se lo agradezco a la clínica.»

El caso de Ana es muy similar al de Josefina, una mujer gallega octogenaria que sigue regentando la que aún hoy es una de las más conocidas confiterías y empresa de catering de Montevideo. Josefina se casó con un marino holandés, tuvo dos hijos y enviudó poco después. Siempre fue la principal sustentadora del hogar.

En general, por lo que respecta al trabajo de campo realizado hasta el momento, la mayor parte de las entrevistadas valoran positivamente su incursión empresarial. Tener un negocio familiar les ha permitido manejar mejor su tiempo en la mayor parte de los casos. Además, la independencia económica les ha permitido tener mayores cuotas de poder en la negociación dentro del ámbito familiar. Por el contrario, en el caso de los hombres empresarios entrevistados, se observan modelos de conciliación familiar más cercanos al patriarcalismo.

\section{Conclusiones}

Podemos analizar la imbricación de la dimensión familiar en las empresas familiares de inmigrantes en tres dimensiones: como recurso de clase, como recurso étnico y en el marco de la conciliación de la mujer empresaria o trabajadora en la empresa familiar. Estos tres aspectos se van combinando en los diferentes momentos de la empresa: nacimiento, desarrollo y madurez. La utilización de los recursos de clase en la familia adquiere mayor relevancia en la fase inicial de la empresa y en la sucesión. El recurso a la solidaridad familiar para llevar a cabo el proyecto empresarial tiene más fuerza en los primeros momentos de la empresa, así como en las etapas en que el negocio entra en crisis. Por último, la inserción laboral de las mujeres en la empresa familiar forma parte de una posible estrategia para conciliar vida familiar y laboral; la familia, en este sentido, influye tanto en la toma de la decisión de emprender un negocio como en su discurrir cotidiano. La triple dimensión que alcanza el aspecto familiar parece confirmarse en las entrevistas realizadas.

Una de las conclusiones que se pueden destacar a la luz del trabajo de campo es que, si bien estas empresas se diferencian de la empresa familiar gallega en Montevideo, fundamentalmente, por el origen del emprendedor que la constituye y los aspectos contextuales (diferentes estructuras familiares, diferencias culturales, distintos contextos institucionales, etc.), hay muchos puntos de unión con las empresas familiares de autóctonos. Por otra parte, aunque la propiedad de la empresa esté en manos de personas inmigrantes, las empresas estudiadas no responden a patrones ideales étni- 
cos (Kloosterman y Rath, 2001), aunque sí es cierto que se identifican muchos rasgos distintivos de este tipo de comercios.

Los factores específicos de este tipo de empresas dirigidas por inmigrantes están determinados, en primer lugar, por elementos de tipo cultural (Light, 1972) — como el tipo de familia - y, en segundo lugar, por el contexto social, político y económico en el país de destino (Kloosterman, 2000).

Los elementos de carácter cultural que caracterizan a este tipo de empresas hacen referencia, sobre todo, a la dimensión familiar, puesto que también la dimensión familiar es la característica más importante de una empresa familiar. Las diferencias con la empresa familiar autóctona se deben a que la sociedad de origen del individuo se puede plasmar en modelos de familia diferentes. Dentro de los modelos de familia, el aspecto que cobra más peso al intentar dilucidar las implicaciones entre empresa y familia es la división sexual del trabajo.

Por otra parte, los modelos de familia no son una realidad abstracta, y tampoco se puede relacionar a priori un modelo determinado de familia con una nacionalidad. En el caso de las familias inmigrantes que tienen un negocio, su modelo de división sexual del trabajo tampoco es permanente, sino que se va modificando. Además, se deben tener en cuenta las alteraciones añadidas que supone el hecho migratorio y el grado de integración con el modelo familiar del entorno en la sociedad de acogida.

Los empresarios estudiados rompen con el «tipo ideal» de inmigrante emprendedor que se define en algunos estudios de negocios étnicos desarrollados en otras partes de Europa y en Estados Unidos (Kloosterman, 2000; Kloosterman y Rath, 2001; Waldinger, Aldrich y Ward, 1990; Light y Gold, 2000; Light, 1972; Light y Roach, 1996). Para algunos autores, el empresariado inmigrante se inserta en la frontera de la economía legal e ilegal, creando pequeños negocios que no requieren una inversión inicial elevada. Sin embargo, en el caso de las empresas estudiadas en el trabajo de campo, se trata de negocios que están integrados en la economía autóctona de manera normalizada.

Otra característica que señala la literatura anglosajona de los negocios étnicos es el bajo nivel de instrucción de los inmigrantes que deciden llevar a cabo una incursión empresarial. Por el contrario, en la muestra estudiada, la mayoría de los emprendedores tienen un nivel de estudios y un nivel cultural elevado; algunos incluso poseen estudios universitarios. En su sociedad de origen pertenecían a la clase media y, en la sociedad de acogida, han «recuperado» su antiguo estatus mediante la empresarización.

La utilización de la familia como recurso de clase es muy explícita en algunos casos estudiados. Por tanto, parece que se confirma la presunción de la importancia de la tradición empresarial familiar como un factor decisivo en la empresarización en la sociedad de acogida. Por lo que respecta a la familia como recurso étnico, podemos 
comentar, a tenor de los resultados, que el recurso a la mano de obra familiar de forma «solidaria» es una consecuencia de la necesidad de poder competir en el mercado general. De nuevo, el recurso a la familia no difiere del recurso a la red familiar que ejercen las empresas familiares de autóctonos sino por el modelo de familia y la división sexual del trabajo.

Las mujeres empresarias con cargas familiares que se han entrevistado se diferencian de las mujeres empresarias autóctonas en que, en muchos casos, no poseen una red familiar extensa en la sociedad de acogida que les permita distribuir la carga de las tareas reproductivas. Para ellas, la flexibilidad horaria de que disponen al ser empresarias es una ventaja. Por otra parte, estas mujeres inmigradas tampoco tienen el mismo nivel de acceso a las políticas sociales que las mujeres autóctonas (Parella y Samper, 2007). Estos dos elementos — red familiar y políticas sociales - determinan estrategias de conciliación tradicionales y patriarcalistas que se perpetúan en la estructura de la empresa familiar.

En esta aproximación a la situación de las empresas familiares de inmigrantes se puede constatar que existe una categoría diferenciada en las empresas familiares y en los negocios étnicos compuesta por las empresas familiares de inmigrantes. Estas empresas, por una parte, se distinguen de las empresas familiares de autóctonos, puesto que las personas que las crean y las componen se hallan inmersas en un proceso migratorio. Por otra parte, también difieren de los negocios étnicos, puesto que no son empresas donde destaquen las características étnicas que definen a este tipo de empresarización en la literatura existente.

\section{Bibliografía}

Alberdi, I. (1996). «Parsons. El funcionalismo y la idealización de la división sexual del trabajo», en Durán, M. A. Mujeres y hombres en la formación de la teoría sociológica. Madrid: CIS.

Anthias, F. (2000). Gender and migration in Southern Europe: women on the move. Berg: Oxford University Press.

Aparicio, R.; Tornos, A. (2000). La inmigración y la economía española. Madrid: Ministerio de Trabajo y Asuntos Sociales. Instituto de Migraciones y Servicios Sociales.

Aranda Gallego, J. (2003). «La inmigración en España: consideraciones económicas e incidencia en el sector financiero español», Papeles de Economía Española, 98: $147-164$. 
Barrada, A. (2003). «Inmigración y seguridad social», Papeles de Economía Española, 98: 121- 145 .

Barret, G.; Jones, T.; McEvoy, D. (2001). «Socio-economic and policy dimensions of the mixed embeddedness of ethnic minority business in Britain», Journal of Ethnic and Migration Studies, 27:241-258.

Beck-Gernsheim, E. (2003). «Contrato generacional y relación entre sexos», en La reinvención de la familia. En busca de nuevas formas de convivencia. Barcelona: Paidós.

Beck-Gernsheim, E. (2003). La reinvención de la familia. En busca de nuevas formas de convivencia. Barcelona: Paidós.

Brunet, I, Alarcón, A. (2004). «Mercado de trabajo y familia», Revista de Investigaciones Políticas y Sociológicas, 4 (2): 115-129.

Carrasco, R. (2003). «Inmigración y mercado laboral», Papeles de Economía Española, 98: 94-108.

Castles, S.; Kosack, G. (1984). Los trabajadores inmigrantes y la estructura de clases en la Europa Occidental. México: Fondo de Cultura Económica.

Curran, S.; Shafer, S.; Donato, K. M.; Garip, F. (2006). «Mapping Gender and Migration in Sociological Scholarship: Is It Segregation or Integration?», International Migration Review, 40 (1): 199-223.

Engelen, E. (2001). «'Breaking in' and 'breaking out': a Weberian approach to entrepreneurial opportunities», Journal of Ethnic and Migration Studies, 27 (2): 203223.

Flaquer, L. (1998). El destino de la familia. Barcelona: Ariel.

Gallo, M. A. (1998). La successió a l'empresa familiar. Barcelona: Servei d'Estudis de La Caixa.

Gallo, M. A.; Cuatrecasas, E*; Arriola, J. M.; Casado, F* (1998). La empresa familiar. Donostia-San Sebastián: Asociación de Empresarios de Gipuzkoa.

Green y Johns (1973). Introducción a la sociología. Buenos Aires: Labor.

IoÉ, Colectivo (2002). Inmigración, escuela y mercado de trabajo. Barcelona: Fundación La Caixa.

Kloosterman, R. (2000). «Immigrant Entrepreneurship and the Institutional Context», en Rath, J. Immigrant Businesses. The economic, political and social enviroment. University of Warrick and Centre for Research in Ethnic Relations.

Kloosterman, R.; Rath, J. (2001). "Immigrant entrepeneurs in advanced economies: mixed embeddedness further explored», Journal of Ethnic and Migration Studies, 27: 189-201.

Kloosterman, R.; Van Der Leun, J*; Rath, J. (1999). «Mixed Embeddedness: (In)formal Economic Activities and Immigrant businesses in the Netherlands», International Journal of Urban and Regional Research, 23 (2)ः 253-267. 
Lagrave, R. M. (1993). «Una emancipación bajo tutela. Educación y trabajo de las mujeres en el siglo XX», en Duby, G. y Perrot, M. Historias de las mujeres. Madrid: Taurus.

Light, I.; Gold, S. (2000). Ethnic Economies. San Diego: Academic Press.

Light, I;; Roach, E. (1996). «Self-Employment; Mobility Ladder or Economic Lifeboat?», en Waldigner R.y Bozorgmenr, M. Ethnic. Los Ángeles: Russell Sage Foundation.

Light, I. (1972). Ethnic Enterprise in America. Business and Welfare Among Chinese, Japanese and Blacks. California: University of California Press.

Murillo, S. (1996). «El liberalismo: el derecho a la igualdad versus privacidad», en El mito de la vida privada. Madrid: Siglo XXI.

Osborne, R. (2004). «Del padre simbólico al padre real: la función paterna desde la modernidad», en Osborne, A. y De la Concha, R. Las mujeres y los niños primero. Discursos sobre la maternidad. Barcelona: Icaria.

Parella, S. (2003). «Repensando la participación de las mujeres en el desarrollo desde una perspectiva de género». Papers. Revista de Sociologia, 69: 31-57.

Parella, S. (2003). Mujer, inmigrante y trabajadora: la triple discriminación. Barcelona: Antrophos.

Parella, S.; Samper, S. (2007). «Factores explicativos de las percepciones y estrategias de conciliación del ámbito laboral y familiar de las mujeres inmigradas», Papers. Revista de Sociologia [en prensa].

Parsons, T. (1982). El sistema social. Madrid: Alianza Editorial.

Sassen, S. (1991). The global city: New York, London, Tokyo. Princeton: Princeton University Press.

SASSEN, S. (1993). La movilidad del trabajo y del capital. Un estudio sobre la corriente internacional de la inversión y del trabajo. Madrid: Ministerio de Trabajo y Seguridad Social.

Sinke,S.M.(2006) «Gender and Migration: Historical Perspectives», International Migration Review, 40 (1): 82-103.

Solé, C.; Parella, S. (2005). Negocios étnicos. Los comercios de los inmigrantes no comunitarios en Catalunya. Barcelona: Fundació CIDOB.

Thumerelle, P. J. (1997). Las poblaciones del mundo. Madrid: Cátedra.

Waldinger, R., Aldrich, H.; Ward, R. (1990). Ethnic Entrepreneurs. California: Sage.

Wright, R.; Ellis, M. (2001). «Immigrants, the Native- Born, and the Changing Division of Labor in New York City», en Forner, N. New Immigrants in New York. New York: Columbia University Press. 
